Disability and Rehabilitation

\title{
Partners towards autonomy: risky choices and relational autonomy in rehabilitation care
}

\section{Matthew R. Hunt \& Carolyn Ells}

To cite this article: Matthew R. Hunt \& Carolyn Ells (2011) Partners towards autonomy: risky choices and relational autonomy in rehabilitation care, Disability and Rehabilitation, 33:11, 961-967, DOI: $10.3109 / 09638288.2010 .515703$

To link to this article: https://doi.org/10.3109/09638288.2010.515703

曲 Published online: 22 Sep 2011.

Submit your article to this journal $\pi$

Џll Article views: 1179

Q View related articles $\sqsubset$

Citing articles: 22 View citing articles 


\title{
Partners towards autonomy: risky choices and relational autonomy in rehabilitation care
}

\author{
MATTHEW R. HUNT ${ }^{1,2}$ \& CAROLYN ELLS ${ }^{3,4}$ \\ ${ }^{1}$ Centre for Research on Ethics (CRÉUM), University of Montreal, Montreal, Quebec, Canada, ${ }^{2}$ Department of Clinical \\ Epidemiology and Biostatistics, McMaster University, Hamilton, Ontario, Canada, ${ }^{3}$ Department of Medicine, McGill \\ University, Montreal, Quebec, Canada, and ${ }^{4}$ Sir Mortimer B. Davis fewish General Hospital, Montreal, Quebec, Canada
}

Accepted August 2010

\begin{abstract}
Purpose. A common source of ethical challenge for health care professionals (HCPs) in rehabilitation is situations when patients wish to make risky choices not related or contrary to rehabilitation goals. We explore the potential contribution of a relational understanding of autonomy for orienting clinical practice when patients wish to enact choices with associated risks for the patient or others.

Method. We provide a theoretical analysis that is oriented by an examination of risk and a relational conception of autonomy, as relevant to rehabilitation care. We illustrate our analysis through the examination of a clinical case.

Results. Relational autonomy assumes that the patient, and the patient's decisional autonomy, is situated and shaped by relationships. From this perspective, HCPs can engage in a process of communication and deliberation with the patient about the risky choices at issue, leading towards improved patient autonomy.

Conclusions. Relational autonomy can contribute to understanding patients' risky choices and guiding HCPs as they partner with patients towards autonomy. Such an approach supports patient-centred rehabilitation care. Ultimately, as clinicians respond to a patient who wishes to enact a risky choice, they should aim for a partnership towards autonomy with the patient and family.
\end{abstract}

Keywords: Risk, ethics, autonomy, relational autonomy

\section{Introduction}

Key goals of rehabilitation are the promotion of functional independence, support of professional and social reintegration, and development of compensatory capabilities to respond to the consequences of injury, disease or disability. Addressing these goals requires that patients attempt new skills and push the boundaries of their capacities, which involves risk. Risks associated with rehabilitation care can be manifested as risk of harmful consequences of therapeutic interventions, or risk of failure and discouragement [1]. In this article, we address a different kind of risk that enters into rehabilitation care with some frequency: choices that patients wish to make that are not guided by rehabilitation-oriented goals, and that entail risk. Such choices may result in risks for the patient or for others.

Ethical concerns reported in the rehabilitation literature include concerns about risks associated with choices and actions that appear counter to rehabilitation goals [2]. In a study of health care professionals (HCPs) working in a rehabilitation hospital in British Columbia, Canada, Young and Sullivan identified the following common ethical concerns: (1) tension between patient autonomy and professional autonomy, (2) risk taking behaviours of patients and (3) disruptive, uncooperative or aggressive behaviour [3]. In a survey of HCPs in a rehabilitation hospital in Chicago, Illinois, Kirschner et al. found that 'rehabilitation professionals questioned the limits to their responsibility when patients 
and families refused their recommendations for 'safe' discharge plans, or when resources were not available to provide the care needed' [4, p. S7]. Consistent with empirical research examining ethics in rehabilitation practice, one of us $(\mathrm{MH})$ is a clinical ethics consultant at a rehabilitation hospital where risk is frequently an important facet of ethics questions posed by clinicians. While our focus in this article is on HCPs, we note that non-professionals involved in the care of patients (e.g. orderlies, nurse assistants, physiotherapy technicians, etc.) may also feel challenged when called upon to assist in (or witness) risky activity that appears contrary to rehabilitation goals.

Addressing situations when patients wish to take actions that entail risks not related to the goals of care (at least as understood by HCPs) is rendered more complex due to uncertainties associated with risk evaluation, differences in how risks are perceived and challenges associated with communication about risk. A key consideration in thinking about risk is the inherent uncertainty regarding evaluations of risk [5]. HCPs may judge an action or choice to be risky, but in many cases the actual likelihood of harm is difficult to predict. Quantitative assessments and empirically generated estimates are not available for many risks that arise in rehabilitation. Assumptions of HCPs also enter into estimations of risk. HCPs may differ in how they perceive the risks associated with a particular situation. This is not surprising as the perception of risk differs between individuals based on many factors including their conceptions of risk, hazard domains, publicity, past experiences, fears, personal values and worldview [6]. Because in many cases patients and HCPs will perceive risks differently, it is useful to distinguish risks that are supported by clinical data and evidence and risks that are linked to judgments and assumptions that have yet to be evaluated or tested. While the critical role and value of clinical judgment should be acknowledged, the degree of confidence (whether strong or weak) regarding estimations of risk should be taken into account, and factored into risk assessment and management. An additional challenge of risk assessment relates to communication. Risk is a notoriously difficult concept to communicate [5,7]. HCPs need to account for these challenges and seek to ensure effective communication with patients, families and other staff members regarding risk.

HCPs understandably may feel anxious when, for example, a dysphagic patient refuses the modified diet that has been prescribed to decrease the risk of aspiration and pneumonia, a post-operative patient disregards weight-bearing restrictions and foregoes the use of crutches, or a patient who has sustained a mild traumatic brain injury insists on being discharged to an environment that the health care team believes to be unsafe for her. These examples represent patient choices that increase risks primarily to the patient her or himself. Other choices that entail risks for the patient also pose risks for others, including family members, HCPs or other third parties. For example, a patient with a history of accidentally starting fires in his kitchen wishes to return to live alone in his apartment; a patient whose judgment is impaired plans to return to her job as a school crosswalk guard, or a patient with life-long dare-devil tendencies repeatedly drives his electric wheelchair in a reckless fashion through the halls of the rehabilitation hospital.

Actions that involve heightened risk may be seen as working against HCPs' desire to provide optimal care for their patients and hope for the best outcomes possible for their rehabilitation. Further, HCPs may question what their professional obligations require. It is reasonable to interpret that applicable professional obligations lead to opposing courses of action. For example, the commitments of HCPs include promoting patients' wellbeing, protecting them from harm and supporting their autonomous choices. By helping patients to act on their risky choices that are unaligned with rehabilitation goals, HCPs would seem to show respect for their patients' autonomy, but with the consequence of risking rather than promoting their patients' wellbeing. In contrast, to hinder patients from enacting such choices may protect patient safety, but at a cost of interfering with their autonomy. Similarly, professional duties to third parties such as to patients collectively, future patients, colleagues, their profession, members of the public and society as a whole may be compromised. Being implicated in patients' risky choices may also pose a challenge to HCPs' professional autonomy to act within the standards of their profession. Furthermore, some may feel guided by their professional role in one direction (i.e. to support or not support patients' risky choices), but as a citizen or fellow human they may feel guided in another incompatible way. In either case, typically the principle of respect for patient autonomy is implicated.

We propose that some of these tensions experienced by clinicians can be mitigated by taking a broad, nuanced view of autonomy and the ethical principle of respect for autonomy. While other ethical considerations (e.g. beneficent care, reduction of harms, ethical judgement, other important duties of HCPs) are relevant and remain to be addressed, in this article we start (and respond) where we believe HCPs experience some of the greatest tension (i.e. what respect for patient autonomy means and requires). In so doing, we hope to leverage HCPs over a common and troubling hurdle involving patients' risky choices in rehabilitation care. Drawing from recent scholarship on 
autonomy to inform our analysis, we describe and illustrate how a relational conception of autonomy can provide a valuable framework and useful vocabulary for orienting clinical practice. We then use a narrative analysis to illustrate how relational autonomy can orient HCPs as they work with patients who wish to make risky choices that are contrary to (or outside) the rehabilitation goals that guide HCPs.

\section{Relational autonomy}

The core concept of autonomy involves having independence from controlling influences and the capacity to act with intention [8]. There are numerous applications of this concept within different domains (e.g. someone with 'financial autonomy' has independence and capacity to act with intention regarding their own financial matters, with 'religious autonomy' the domain of one's autonomy is one's religious beliefs and practices). In the context of rehabilitation, 'autonomy' has two important usages. First, autonomy is used to describe an individual's functional independence, capacity for self-care and relative freedom from disability. In this work, we focus on a second usage of autonomy which is typically construed as an individual's capacity to make informed, intentional choices, without controlling influences, in areas related to the goals and preferences they set out for themselves and their lives [8]. Autonomy in this second sense is assumed to be something particularly unique and valued about humans, which is why ethical and legal norms so often require that it be respected. This is especially the case in healthcare where ethical and legal norms require a patient's informed consent prior to any intervention upon the patient by HCPs.

It is important that policies and practices that define norms for autonomy and respect for autonomy accord with the real lives of people and the healthcare context. Thus for choices about healthcare 'for an action to qualify as autonomous it needs only a substantial degree of understanding and freedom from constraint' [8, p. 101]. Accordingly, in rehabilitation contexts, patient autonomy is often assumed to be respected in situations of discrete patient choices where HCPs provide patients with sufficient information and ensure that patients' substantially understand that information, choose voluntarily and have the necessary capacity to make the particular choice in question.

If one focuses too simplistically or narrowly on discrete instances of informed consent about treatment decisions as the way to respect autonomy, one may see some obvious challenges in applying the principle in practice. For instance in rehabilitation care, a patient's capacity for decision-making may be (temporarily or permanently) damaged, underdeveloped, diminished, or fluctuating. It can be difficult for patients to appreciate possibilities or assess benefits and burdens soon after experiencing a traumatic injury or new disability. Patients in rehabilitation frequently grapple with personal identity issues, such as an identity in flux or being reconstructed. In the wake of a crisis experience, they may reassess and possibly change their values or how these will be applied in new circumstances $[9,10]$. Where consistency over time is often an indicator that gives HCPs confidence that patients' choices are autonomous, lack of consistency - in values, goals, understanding, character - is common during rehabilitation and possibly desirable for short-term and long-term coping and accommodation to a change in life circumstance.

What is relevant to patients changes as their sense of self and goals change through different phases of recovery. It may take time and reflection before patients with a new traumatic injury or new disability can see recommendations (e.g. to learn to use adaptive equipment) from clinicians as opportunities (e.g. for adjusting to life with impairment). For rehabilitation patients themselves and HCPs, these factors challenge efforts to optimise the information and deliberation needed to form and justify meaningful decisions. Further, if HCPs focus too simplistically or narrowly on discrete choice situations, what happens between or behind specific choices (e.g. how the context shapes choices, how reasoning skills, self-confidence, relationships and other factors that affect choice-making are fostered or not) may be ignored [11].

An important body of scholarship has developed articulating alternate conceptions of the principle of respect for autonomy that account for these questions and concerns $[9,12-16]$. Relational autonomy is a prominent example of this literature, and we draw on it in our analysis. As such, the concept of 'relation' has important applications in our analysis. By 'relation' we refer to the connection and effects of connection among people and how they are situated in the world around them. In the everyday reality of our lives, relations are indefinite sets of multiple connections (like a network) that inform and shape what is connected. For example, patients' experiences and choices may be informed and shaped, either directly or indirectly, by their relations to clinicians, legal and regulatory factors that bear on rehabilitation care, the rehabilitation setting, usual daily life routines and commitments, sense of identity, goals, relationships with others, their particular disability/impairment, and more. We use the adjective 'relational' (as in 'relational autonomy') to explicitly emphasise our understanding that what 
is referred to (e.g. 'autonomy') is importantly informed and shaped by relations. Acknowledging people, autonomy and rehabilitation care as relational provides a useful stance to assess clusters of factors that inform and shape decision-making involving patients and HCPs.

Importantly, a relational conception of autonomy is based on an understanding of persons as interconnected and situated, and not as isolated rational thinkers. The context and influences that shape options, choices and relationships are taken into account in evaluating the patient's reality.

This analysis leads us to the view that the conception of decisional autonomy that is most frequently enacted in healthcare generally, and rehabilitation in particular, needs to better account for these features of patients. An idealised, impractical view of autonomy will not provide the conceptual resources to evaluate the ethics of risky patient choices. What is required is a realistic approach to autonomy (i.e. practicable and resonate with what we know of patients and the rehabilitation environment). In a relational autonomy approach, the process of seeking informed consent remains relevant and necessary for key moments in the rehabilitation process. However, these choice moments are no longer the entire ambit of the principle of respect for autonomy. Supporting autonomy is still relevant between these distinct decision-points. As Roter also concludes, 'personal, responsive, and fulfilling communication between patients and physicians .... [are key] to help nurture and develop the capacity for meaningful autonomy and sensitive and respectful medical care' [11, p. 23-24].

A relational approach to autonomy is consistent with a shared responsibility among the patient and HCPs (and, potentially, family members and others close to the patient), for shaping and promoting the autonomy of the patient. This shared responsibility entails a partnership amongst these individuals that continues between consent 'choices' [11]. In this view, the principle of respect for autonomy means more than it often is assumed to. The focus is expanded from the discrete 'choice' of a particular moment (and informed consent) to relations and an understanding of respect for autonomy rooted in partnerships.

\section{Implications of relational autonomy for risky choices}

Respect for autonomy, understood relationally, has multiple implications for assessing risky choices of patients that are contrary or outside rehabilitation goals. Rather than simply acquiescing to any choice of a duly informed, competent patient, relational autonomy leads to a focus on partnership, engagement with the particular contextual features that shape and constrain options in a given situation, accounting for power differentials, and understanding of the patient as situated within relationships.

A relational view of autonomy draws attention not only to the particular decision or action being considered, but also to how the decision or action relates to the person's life plan, sense of self, and their socially embedded reality. Engaging with the patient's reality is a key component of patientcentred care [17]. Relational autonomy provides a conceptual account of how the individual is located (i.e. within clusters of relations to previous experiences and to other people) in a patient-centred approach. An important implication of a relational conception of autonomy is the development of partnerships between the patient, family and HCPs. Establishing partnership requires engagement with people's reality, open and attentive communication and the development of trusting, supportive relationships. To achieve these goals HCPs should strive for sharing of information and open, honest exchange with patients, and take the time needed to allow for dialogue and learning (obviously, a challenge given resource constraints in many rehabilitation settings).

In considering choices that involve risk, it is particularly important to probe the meaning the patient associates with the choice that is being considered, as well as to seek to understand the meaning of potential consequences that have been identified with different options, and how these are incorporated in the patient's evaluation of risk. Particular options may be associated with both instrumental and symbolic importance for patients.

HCPs should also consider the origins of their own understanding of particular options. They should consider how assumptions and values that they hold enter into their own evaluation of the identified risk. Patients and HCPs may put different values on the potential consequences of options. HCPs should question themselves and examine how their own values and assumptions related to a particular situation influence their understanding of the choices that are being considered or being made. Practicing moral imagination, attempting to see a situation from the experiential perspective of another person, may also provide valuable insight into a particular situation.

A relational conception of autonomy influences how family members, and others close to the patient, are understood. Patients are not seen as isolated decision-makers, nor are family members merely seen as sources of hands-on support. Family members are understood as potential participants (if acceptable to the patient) in decisionmaking processes. By acknowledging the ways that 
individuals are interdependent with others for support and care in a myriad of practical and emotional ways, attention is given to the embeddedness of the individual within a local social world. This attention can guide clinicians towards the possibility and value of involving others from the patient's family or close entourage to support the patient (for both functional independence and autonomy in decision-making).

A relational conception of autonomy can help HCPs think about how to promote the decisionmaking autonomy of their patients, and share in the process. We further explore these implications of a relational conception of autonomy through the following narrative analysis where HCPs consider their response to a patient's desire to enact a risky choice. Narrative analysis is a useful pedagogical approach to help HCPs develop skills to identify and address ethical issues in everyday practice. While writing and analysing a personal narrative can be especially valuable for HCPs in developing ethical mindfulness in their own practice, here we use the technique to analyse a fictional case and to model the kinds of trigger questions and reflection that HCPs might use [18].

1. Mr Smith is a 68 year old patient who had a right lacunar stroke three months ago. He is nearing the end of a two month stay in an in-patient rehabilitation unit and is adamant that he return to his home soon. The team is concerned about Mr. Smith's upcoming discharge. Mr Smith is a widower and lives alone in a second floor apartment. He ambulates with a walker. He can go up and down stairs independently by sitting on each step. The homecare team has visited his apartment and installed grab bars and a raised toilet seat in his bathroom. A registered nurse is scheduled to visit Mr. Smith three times a week. However, the team is very concerned that Mr. Smith will not be safe living by himself in the apartment.

Discharge to an unsafe location is a common ethical issue arising in rehabilitation practice $[4,19]$. The case reminds us of the different ways that autonomy is relevant to rehabilitation: autonomy as capacity in matters of self-care and functional ability (i.e. functional autonomy), and autonomy as the capacity to make informed, intentional and nonmanipulated choices related to goals and preferences one has for one's life (i.e. decision-making autonomy). Some aspects of Mr. Smith's ability to function independently at home have been addressed through home visit, adaptive equipment and organisation of home care services. Yet is Mr. Smith's adamant desire to return home an unequivocal expression of his autonomous decision-making? To evaluate this question further examination of the meaning that options hold for Mr. Smith, and the contextual and relational features that shape Mr. Smith's reality is necessary.

That an older person who has been living alone prior to a medical event wishes to return home upon discharge is not a surprising preference. Home frequently represents easy access to neighbours, community and familiar services, continuity with one's history and memories, space to follow one's routines and habits, and where one feels most oneself and safe. Nonetheless, Mr. Smith's new reality needs to accommodate somehow the sequelae of his recent stroke.

Communication between $\mathrm{Mr}$. Smith and the treatment team should include exploration of the rationale for Mr. Smith's wishes, as well as the nature of the HCPs' concerns. An essential component of this process is to discuss the meanings $\mathrm{Mr}$. Smith associates with returning home, or with competing options (e.g. What does dependence on others mean for Mr. Smith? What is at stake for him in accepting to move to a supported-living environment?). Drawing attention to the 'why' question is vital to understanding and assessing the situation, as well as building trust in the therapeutic relationship.

As the discussion progresses between Mr. Smith and the members of the rehabilitation team, their shared deliberation should focus on examining which option, or cluster of options, is best for Mr. Smith at this time. In preparation for this discussion, HCPs should work to identify and evaluate possible options. Creativity and imagination may help Mr. Smith and the HCPs identify options and strategies that are not immediately apparent.

At the same time it is important to examine contextual features associated with the decisions that need to be made. As well as Mr. Smith's functional abilities and medical situation, relevant contextual features include the types of formal and informal assistance that are available to him. A critical question to ask is whether the situation of risk results from inadequacies in the health and social services system (e.g. Does the system meet Mr. Smith's needs?). The team can then examine what supports and structures can be accessed both to promote functional autonomy as well as autonomy in decision-making (e.g. Are there groups or resources that $\mathrm{Mr}$. Smith can access while living at home or will his mobility constrain access to potential participation?).

This dialogic approach will lead to a better understanding of risks, and the meaning and context of those risks for Mr. Smith. Furthermore, this exchange promotes partnership between Mr. Smith and the HCPs who work with him as they both share the goal of promoting Mr. Smith's overall well being. This process will help to reveal what each party feels are important considerations and where 
uncertainties, inconsistencies and complexities lie. For example, suppose Mr. Smith wishes to return home where he feels most safe, however HCPs view this option as entailing important risks to safety. Establishing this could lead to further discussion of the nature and experience of feeling and being safe.

2. Mr Smith has two daughters who live in the same city. They have visited their father regularly during his stay in the rehabilitation centre, however both daughters have young families and lead busy lives.

Mr. Smith's daughters first might be seen by HCPs as potential caregivers and sources of support at home. This may indeed be accurate. Given the daughters' regular visits they clearly have a relationship with their father that is a value to them. However, HCPs should explore whether this level of attention is something that the daughters could sustain if the patient moved home. The consequences and risks for the daughters that are associated with the possible options should be taken into account. The involvement of Mr. Smith's daughters also provides opportunities for those involved to better understand the meaning and context of the decisions at hand. Family members can provide valuable insight into Mr. Smith's previous life, as well as helping to imagine future possibilities, and identify forms of assistance and support that may not have been considered.

Including Mr. Smith's daughters in these deliberations may also help to reveal other aspects of the situation. Perhaps Mr. Smith's wife died soon after moving to a nursing home and he felt he had abandoned her there. Or, perhaps while his wife was in the nursing home their grandchildren were reluctant to come to visit her, and Mr. Smith cannot imagine becoming separated from his family in this way. The daughters may be able to provide perspectives that Mr. Smith has been unwilling or unable to acknowledge on his own. Becoming aware of how these factors relate to his current decisionmaking may help Mr. Smith, his family, and the team to identify and prioritise what is most important to him (and compromise on other factors) in planning post-discharge supports.

3. The team is also concerned that Mr. Smith might start a fire while attempting to cook his meals. When tested by the psychologist, Mr. Smith has decreased short-term memory and his problem solving skills appear limited.

Risks in rehabilitation may accrue for others, not just for the patient. If Mr. Smith sets his kitchen afire, both he and his neighbours may be harmed.
Considering third party risks is a challenging issue, and not easily resolved. Part of a relational approach to understanding autonomy is acknowledging that there may be opportunities to promote greater autonomy for those whose autonomy is limited or constrained. For instance, working to improve Mr. Smith's short-term memory and problem solving can be important means of enhancing autonomy for decision-making (in addition, to contributing to functional independence more broadly). Thus, in situations of risk, including risk to third parties, HCPs should not only seek to address the patient's functional capacities (for example, the ability to cook safely) but also their ability to evaluate potential consequences (for example, the risks that an action poses to others).

It is appropriate to discuss with the patient the responsibilities that they have towards others, and to directly address foreseeable risks. Members of the rehabilitation team should have a frank discussion with $\mathrm{Mr}$. Smith to evaluate the risk of Mr. Smith causing a fire in his kitchen. Strategies to minimise the risk and prevent negative consequences should be explored and acted upon were Mr. Smith to move back to his apartment upon discharge. These strategies might include alternative meal arrangements such as meals-on-wheels, or (if the estimation of the risk is high enough) removal of appliances with heating elements from his apartment, while ensuring that Mr. Smith's nutritional needs are met. In approaching these discussions, HCPs should strive for collaboration and consensus. It will also be beneficial to work with $\mathrm{Mr}$. Smith to establish parameters for when he should ask for help (e.g. if certain things happen he should call a particular person for assistance). In evaluating these possibilities, a supervised home visit or weekend pass will allow for the identification of necessary supports, as well as greater clarity regarding the feasibility of particular options.

The existence of risk of serious harm for others flags the HCPs' responsibility to attempt to prevent such harm from occurring. This situation emphasises that respecting autonomy is one of several important ethical concerns. While wanting to maximise autonomy, HCPs also need to attend to the range of important ethical responsibilities and principles. HCPs have responsibilities towards society and the common good, as well as to each individual patient. The nurses who will regularly visit Mr. Smith, as well as family visitors, should be vigilant in evaluating risk on an ongoing basis, and identifying opportunities to reduce the likelihood of risk or the gravity of potential harms. If HCPs have an exclusive focus on merely accepting individual choices (understood as respecting 
individual autonomy) they miss important moral considerations, and act irresponsibly.

\section{Conclusion}

In this article, we consider the challenge for HCPs when patients wish to enact risky choices that are contrary or outside the goals of rehabilitation care. We distinguish several components of risk, risk assessment and risk management and draw on a relational conception of autonomy to help HCPs respond to such situations. In rehabilitation settings, it is important that HCPs foster both functional autonomy and decisionmaking autonomy. While both of these types of autonomy are best understood as relational (i.e. informed and shaped by indefinite sets of dynamic connections among people and the environment), our discussion brings particular attention to the relational nature of decisional autonomy. Such an approach to thinking about patient autonomy provides a lens that aids in bringing a bigger picture into focus. With attention to the particularity of the individual patient (including the patient's sense of identity, goals, history, and social context), HCPs can more easily engage in a process of communication and deliberation with the patient (and possibly others) that results in improved patient autonomy and a patient-centred care plan that all can accept. Such an approach leads towards the development of a 'partnership towards autonomy' between patients and HCPs in rehabilitation settings.

Declaration of interest: The first part of this essay's title, 'Partners towards autonomy,' is drawn from the title of the Code of Ethics of the Jewish Rehabilitation Hospital in Laval, Quebec, Canada. Matthew Hunt is supported by a post-doctoral fellowship from the Canadian Institutes of Health Research. Carolyn Ells was Visiting Scholar in the Department of Bioethics, Dalhousie University during the preparation of this article.

\section{References}

1. Wade D. Adverse effects of rehabilitation: an opportunity to increase quality and effectiveness in rehabilitation. Clin Rehabil 2009;23:387-393.

2. Browne A. Helping residents live at risk. Camb Q Healthc Ethics 2003;12:83-90.

3. Young JM, Sullivan WJ. Ethical concerns of staff in a rehabilitation center. HEC Forum 2001;13:361-367.

4. Kirschner KL, Stocking C, Brady Wagner L, Foye SJ, Siegler M. Ethical issues for rehabilitation clinicians. Arch Phys Med Rehabil 2001;82(Suppl 2):S2-S8.

5. Macciocchi SN, Stringer, AY. Assessing risk and harm: the convergence of ethical and empirical considerations. Arch Phys Med Rehabil 2001;82(Suppl 2):S15-S19.

6. Slovic P. Perception of risk. Science 1987;236:280-285.

7. Alaszewski A, Horlick-Jones T. How can doctors communicate information about risk more effectively? Br Med J 2003; 327:728-731.

8. Beauchamp TL, Childress JF. Principles of biomedical ethics. 6th ed. New York: Oxford University Press; 2009.

9. Friedman M. Autonomy, gender, politics. New York: Oxford University Press; 2003.

10. Bergsma J, Thomasma D. Autonomy and clinical medicine: Renewing the health professional relation with the patient. Dordrecht, The Netherlands: Kluwer; 2000.

11. Roter D. The medical visit context of treatment decisionmaking and the therapeutic relationship. Health Expectations 2000;3:17-25.

12. Kukla R. Conscientious autonomy: displacing decisions in health care. Hastings Center Rep 2005;35:34-44.

13. Donchin A. Understanding autonomy relationally: toward a reconfiguration of bioethical principles. J Med Philos 2001;26: 365-386.

14. Mackenzie, Stoljar N, editors. Relational autonomy: feminist perspectives on autonomy, agency, and the social self. New York: Oxford University Press; 2000.

15. Sherwin S. A relational approach to autonomy in health care. In: Sherwin S, editor. The politics of women's health: exploring agency and autonomy. Philadelphia, PA: Temple University Press; 1998. pp 19-47.

16. Cardol M, De Jong BA, Ward CD. On autonomy and participation in rehabilitation. Disabil Rehabil 2002;24: 970-974.

17. Stewart M. Towards a global definition of patient centered care. Br Med J 2001;322:444-445.

18. Guillemin M, McDougall R, Gillam L. Developing "ethical mindfulness" in continuing professional development in healthcare: Use of a personal narrative approach. Camb Q Healthc Ethics 2009;18:197-208.

19. Moats G, Doble S. Discharge planning with older adults: toward a negotiated model of decision-making. Can J of Occup Ther 2006;73:303-311. 